\title{
Regime de inundação e a regeneração arbórea em duas áreas florestais na planície alagável do alto Rio Paraná
}

\author{
Marcos Paulo da Cruz * \\ João Batista Campos \\ PPG em Ecologia de Ambientes Aquáticos Continentais, \\ Universidade Estadual de Maringá Departamento de Biologia, \\ CEP 87020-900 Maringá - PR, Brasil \\ * Autor para correspondência \\ Cruz-p2003@ig.com.br
}

Submetido em 28/05/2012

Aceito para publicação em 11/06/2013

\section{Resumo}

Foi realizado um estudo em dois fragmentos florestais ( $\mathrm{RF}$ - remanescente florestal; e FL - floresta de 57 anos) na planície de inundação do alto Rio Paraná para testar a influência do regime de inundação sobre a estrutura e composição do estrato de regeneração arbóreo. O levantamento foi realizado com os indivíduos arbóreos agrupados em duas classes de tamanho: classe I, $<10 \mathrm{~cm}$ altura, e classe II, $>10 \mathrm{~cm}$ de altura e circunferência à altura do peito $<15 \mathrm{~cm}$. A área amostral foi de $2.400 \mathrm{~m}^{2} \mathrm{em}$ cada um dos fragmentos, divida em 24 parcelas contíguas de $10 \mathrm{~m}$ x $10 \mathrm{~m}$. Foi obtido também, o tempo de inundação ao qual esteve submetido cada parcela ao longo dos últimos quatro anos. O número de indivíduos por classes de tamanho foi comparado entre as áreas amostrais através de análise de variância, do mesmo modo, testou-se a similaridade florística por meio de DCA, associação das espécies com o tempo de inundação foi realizada através de testes de correlação. Um total de 30 espécies foi encontrado nas duas áreas, com um maior número de indivíduos e de espécies para a classe II nos dois ambientes. O RF apresentou um menor tempo de inundação, sendo constituído predominantemente por espécies que se mostraram negativamente correlacionadas com o tempo de inundação. Já na FL, ocorreram espécies correlacionadas positivamente ao tempo de inundação aos quais as parcelas foram submetidas. Dessa forma, o regime de inundação diferenciado entre ambientes, gera uma heterogeneidade ambiental que contribui para o incremento da diversidade de espécies, haja vista, que essa força seletiva pode influenciar no estabelecimento de plântulas e na sua passagem para maiores classes de tamanho.

Palavras-chave: Classes de tamanho; Cota altimétrica; Estrutura da comunidade; Florestas ribeirinhas

\section{Abstract}

Flooding regime and tree regeneration in two forest areas in the upper Paraná River floodplain. We studied two forest fragments (RF - forest remnant, and FL - forest that was 57 years old) in the upper Paraná River floodplain to test the influence of flooding regime on the structure and composition of tree regeneration. We conducted the survey with trees grouped in two size classes: class I, $<10 \mathrm{~cm}$ tall; and class II, $>10 \mathrm{~cm}$ tall and circumference at breast height $<15 \mathrm{~cm}$. The sample areas were $2,400 \mathrm{~m}^{2}$ in each of the forest fragments, which were divided into 24 plots of $10 \times 10 \mathrm{~m}$. We also obtained the time of inundation that each plot was subjected to over the previous four years. The number of individuals in each size class was compared between the sample areas with an analysis of variance. In the same manner, the correlation of floristic similarity with DCA and association of species with the time of flooding were tested. We found up to 30 species in both sites, 
and there was a larger density of plants and more species for class II in both environments. RF had a shorter flooding duration, which is consistent with the predominance of species that were negatively correlated with flooding duration. Conversely, in the FL, there were more species that were positively correlated to a longer duration of flooding. Thus, the differentiated flooding regime between environments generates a heterogeneous environment that contributes to the increase in species diversity, given the fact that this selective force may influence seedling establishment and its transition to larger size classes.

Key words: Community structure; Elevation; Riparian forests; Size classes

\section{Introdução}

Florestas ripárias são formações vegetacionais que margeiam cursos d'água e apresentam características diretamente relacionadas ao regime de inundação, podendo receber outras diversas denominações, tais como: florestas ribeirinhas, matas ciliares etc. (RODRIGUES, 2001). Essas florestas se destacam pela alta riqueza de espécies e diversidade que apresentam (NILSSON; SVEDMARK, 2002), sendo, portanto, consideradas fundamentais para a conservação dos recursos hídricos, da fauna terrestre e aquática (CAMPOS; SOUZA, 2002; TEIXEIRA et al., 2008; CHAPLA; CAMPOS, 2011).

Eventos de inundação são considerados o principal fator estruturador da vegetação ribeirinha (BUDKE et al., 2007; GIEHL; JARENKOW, 2008). Esses eventos alteram diretamente as propriedades físico-químicas dos solos, através de processos de erosão ou sedimentação de matéria orgânica e nutrientes, que podem influenciar tanto a morfologia de indivíduos, quanto a composição, riqueza, diversidade e distribuição das espécies (JUNK, 1999). Além disso, a estrutura das comunidades pode ser alterada diretamente por meio da magnitude, duração e frequência das inundações (NEIFF et al., 1994).

Os efeitos do hidroperíodo sobre a comunidade vegetal são mediados pela heterogeneidade topográfica. Variações topográficas em escala local conduzem a alterações na intensidade e frequência do alcance da coluna d'água, gerando sítios com diferentes teores de umidade, que além de variar espacialmente, podem ainda sofrer alterações ao longo do tempo (OLIVEIRA-FILHO et al., 1994; TEIXEIRA; ASSIS, 2005; 2009; BUDKE et al., 2007; 2008; TEIXEIRA et al., 2008). As condições de alta umidade afetam a germinação e o recrutamento de indivíduos (LOBO; JOLY, 2000). Além disso, várias espécies de florestas inundáveis não germinam enquanto alagadas ou perdem a viabilidade após a submersão
(FERREIRA; RIBEIRO, 2001). Logo após a germinação, ainda nas primeiras fases de desenvolvimento, os indivíduos são frequentemente submetidos à pressão das condições ambientais locais (FELFILI, 1997). Dessa maneira, inundações periódicas representam um dos fatores determinantes do sucesso da regeneração das espécies nessas condições, e por isso afetando a distribuição espacial da vegetação (RESENDE et al., 2003).

Além disso, as inundações também influenciam diretamente as características físico-químicas do solo (OLIVEIRA-FILHO et al., 1994; DALANESI et al., 2004). As características diferenciadas do solo, determinadas pelas diferentes condições de drenagem, resultam em ambientes com diferenças nítidas quanto à estrutura e composição da vegetação. Umetsu et al. (2011) demonstraram que a frequência e o tempo de alagamento foram os principais determinantes da distribuição das espécies vegetais ao longo de um perfil topográfico. Resultados semelhantes foram obtidos por Prata et al. (2011), que encontraram micro-ambientes distintos quanto à composição e estrutura da comunidade arbórea, associados principalmente às diferentes condições de drenagem e alagamento das parcelas. Diversos trabalhos têm demonstrado que as características estruturais da vegetação encontram-se associadas ao regime hídrico e às condições topográficas (OLIVEIRA-FILHO et al., 1994; FERREIRA; STOHLGREN, 1999; DURIGAN et al., 2000; CHAPIN et al., 2002; DALANESI et al., 2004; DAMASCENO-JUNIOR et al., 2005; BUDKE et al., 2007; GIEHL; JARENKOW, 2008; TEIXEIRA; ASSIS, 2009; BIANCHINI et al., 2010).

Dado o entendimento de que a regeneração representa o mecanismo responsável pela manutenção da diversidade dentro dos ecossistemas florestais, entender como esse componente da vegetação se relaciona com as variáveis ambientais em escala local é de grande importância para definir medidas de restauração e conservação das florestas tropicais aluviais. Nesse contexto, o presente trabalho 
teve por objetivo descrever a estrutura e composição da regeneração em dois fragmentos florestais, na planície de inundação do alto rio Paraná e verificar se existe influência dos eventos de inundação nesse processo.

\section{Material e Métodos}

\section{Área de estudo}

O presente trabalho foi desenvolvido em dois trechos de florestas, localizados na Planície de Inundação do Alto Rio Paraná. Uma das referidas áreas é uma floresta de 57 anos (FL) que se desenvolveu sobre uma barra de coalescência fundida à Ilha Porto Rico em 1953 (CAMPOS; SOUZA 2002). Essa área está localizada no município de Porto Rico, Paraná, a $53^{\circ} 17^{\prime} \mathrm{W}, 22^{\circ} 45^{\prime} \mathrm{S}$ e altitude em torno de $232 \mathrm{~m}$. A segunda área é um remanescente florestal (RF), cuja idade não pode ser definida, mas de acordo com fotografias aéreas históricas, em 1952 apresentava vegetação intacta e não há registros de muitos impactos nos anos subsequentes. Essa área está localizada na margem direita do Rio Paraná, Mato

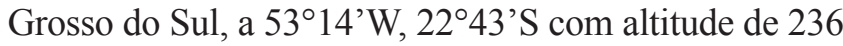
m (Figura 1).

FIGURA 1: Área de estudo - Planície de inundação do alto rio Paraná. A: remanescente florestal (RF); B: floresta de 57 anos (FL).

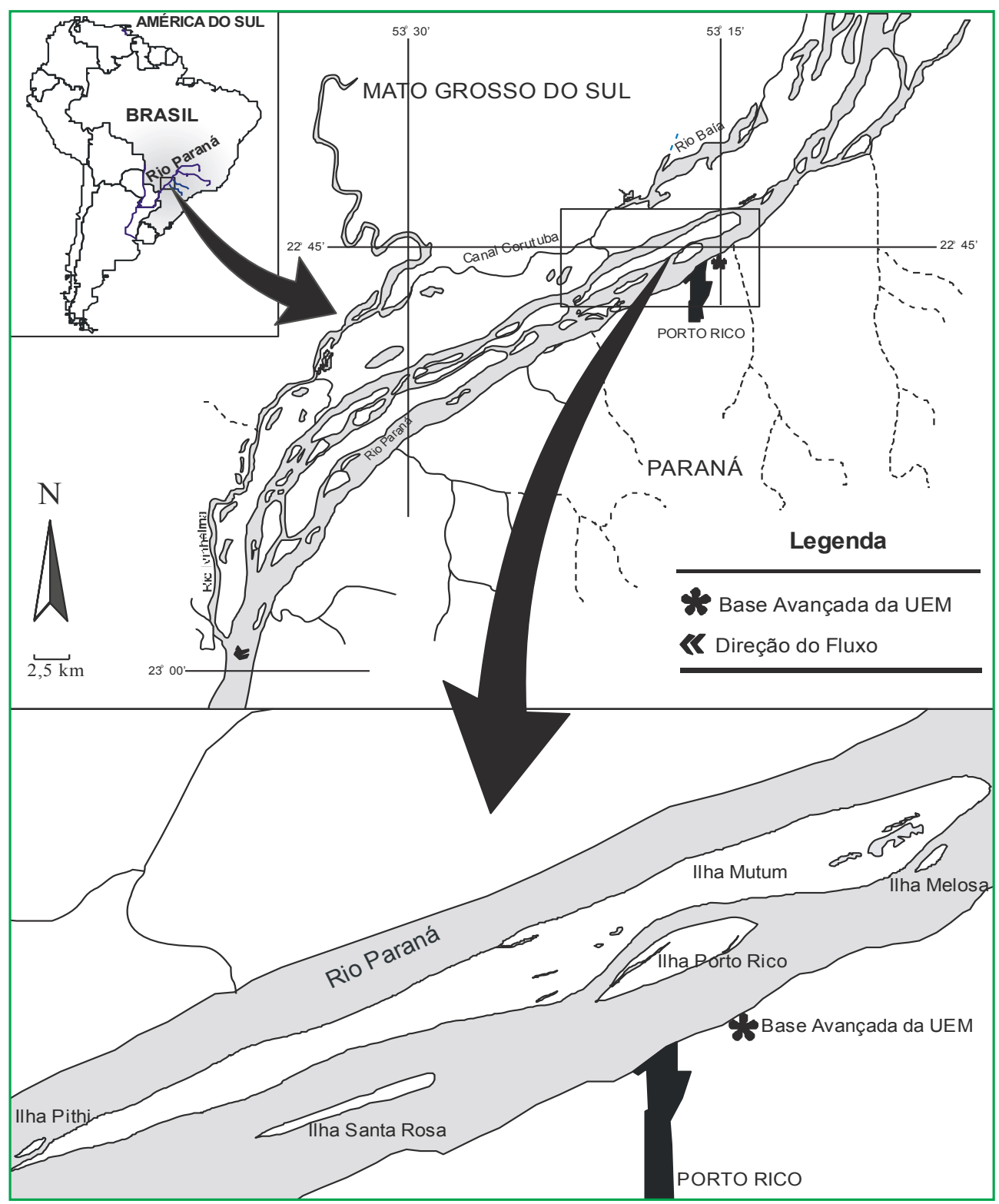


A vegetação dessa região é classificada como Floresta Estacional Semidecidual Aluvial e está inserida no bioma Mata Atlântica. O clima, de acordo com a classificação de Köppen é do tipo Cfa (sem estação seca definida), com temperatura média anual de $22^{\circ} \mathrm{C}$ e precipitação média anual de $1500 \mathrm{~mm}$ (MAACK, 2002).

\section{Delineamento amostral}

Em cada área (RF e FL) foi delimitada uma unidade amostral de $60 \mathrm{~m} \times 40 \mathrm{~m}$ dividida em 24 parcelas contíguas de $10 \mathrm{~m} \times 10 \mathrm{~m}$. A unidade amostral da FL foi alocada com o lado maior paralelo a um Ressaco (corpo aquático de águas lênticas com ligação direta ao Rio) que corta a ilha, com distância de aproximadamente 15 $m$ deste, e a unidade amostral do RF foi disposta com uma configuração semelhante, porém o lado de maior tamanho foi disposto paralelo a uma lagoa natural. As parcelas de $10 \mathrm{~m} \times 10 \mathrm{~m}$ foram utilizadas somente para levantamento de dados topográficos, de modo que foi sorteado um dos vértices de cada parcela para instalação de sub-parcelas de $3 \mathrm{~m} \times 3 \mathrm{~m}$ para levantamento de dados da vegetação.

\section{Levantamento da vegetação}

Dentro de cada sub-parcela foi realizado o levantamento dos indivíduos no estrato de regeneração arbóreo. A definição do termo regeneração é um tanto subjetiva, com a aplicação de critérios de inclusão dos indivíduos de maneira amplamente variável (RONDON NETO, 1999; MARQUES, 2002; OLIVEIRA; FELFILI, 2005). Nesse estudo o estrato de regeneração foi dividido em duas classes de tamanho: Classe I (incluindo plântulas arbóreas com tamanho máximo de $10 \mathrm{~cm}$ ) e classe II (considerando todos os indivíduos arbóreos com mais de $10 \mathrm{~cm}$, até aqueles com circunferência máxima de $15 \mathrm{~cm}$ a $1,30 \mathrm{~m}$ do solo). O material foi identificado no campo e por meio de consulta a literatura especializada.

\section{Dados ambientais}

As parcelas de $10 \mathrm{~m} \times 10 \mathrm{~m}$ foram usadas como referência para o levantamento topográfico que foi realizado utilizando-se nível de mangueira e régua graduada, sendo mensurado a diferença vertical do nível para cada um dos quatro vértices de cada parcela (elevação em relação ao nível do rio). Posteriormente foi estimada a cota média para cada parcela (obtidas através da média das distâncias verticais). A partir dos dados de cota elevacional e dos registros de variação dos níveis fluviométricos da região entre os anos de 2005 a 2008 (Núcleo de Pesquisas em Limnologia, Ictiologia e Aquicultura-NUPELIA,), pode-se estimar a frequência e o tempo de inundação em dias, a que foi submetido cada parcela das duas áreas amostrais.

\section{Análise dos dados}

Para expressar a diversidade de espécies do estrato de regeneração arbórea, foram calculados o índice de diversidade de Shannon (H') e o índice de equabilidade de Pielou ( $\left.\mathrm{J}^{\prime}\right)$. Os valores de $\left(\mathrm{H}^{\prime}\right)$ de cada área amostral e de cada classe de tamanho foram comparados pelo teste $t$ de Hutcheson. A riqueza de espécies das áreas amostrais e de cada classe de tamanho foram comparadas através de curvas de rarefação. O número de indivíduos de cada área e de cada classe foi comparado por meio de análises de variância, seguido por testes pareados de Tukey-Kramer.

As relações de similaridade florística entre as áreas amostrais foram analisadas através de análises de correspondência destendenciada (DCA), calculadas a partir de matrizes de abundância de espécies por parcela. Foram realizados ainda, testes de correlação de Spearman para verificar a associação de cada espécie com o tempo de inundação das parcelas.

\section{Resultados}

O RF apresentou um tempo de inundação com média de $\sim 66$ dias e desvio padrão de $\sim 3$, ao longo dos quatro anos. As parcelas com menor e maior tempo de inundação nesse ambiente estiveram sob condições de alagamento durante 61 e 72 dias respectivamente. Na FLT, o tempo de inundação médio foi de $\sim 172$ dias com desvio padrão de $\sim 35$, durante o mesmo intervalo de tempo. A parcela que permaneceu um menor tempo alagada nessa área, apresentou um tempo de inundação 
de 117 dias e a com maior tempo de inundação esteve alagada em 241 dias. O tempo de inundação entre as duas áreas diferiu significativamente $(\mathrm{F}=225 ; \mathrm{p}<0,001)$.

Encontramos um total de 1570 indivíduos, distribuídos em 30 espécies. Desse total, 1022 indivíduos, representantes de 22 espécies, foram amostrados no RF. Nessa área amostral, a classe de tamanho I somou 169 indivíduos e nove espécies, e a classe II 853 indivíduos e 22 espécies. Na FL, foram amostrados 548 indivíduos e 22 espécies, sendo que a classe I somou 168 indivíduos e cinco espécies e a classe II, 380 indivíduos e 21 espécies (Tabela 1). O número de indivíduos da classe I não diferiu entre as duas áreas $(\mathrm{F}<0,001 ; \mathrm{p}=0,989)$. No entanto, o número de indivíduos da classe II apresentou diferença significativa entre os dois ambientes ( $\mathrm{F}=$ $22,11 ; \mathrm{p}<0,001)$.

No RFID, as espécies mais abundantes na classe I foram Eugenia hyemalis e Albizzia hasslerii, (totalizando $\sim 80 \%$ dos indivíduos), e na classe II, Unonopsis lindmanii, Coussarea platyphylla, Garcynia gardneriana, Eugenia egensis e Eugenia hyemalis, ( $\sim 55 \%$ dos indivíduos). Na FL, as espécies mais abundantes na classe I foram Albizzia hasslerii, Cecropia pachystachya e Celtis iguanaea, (somando 75\%

TABELA 1: Número de indivíduos de cada espécie por classe de tamanho em cada uma das áreas amostrais. RF remanescente florestal; $\mathrm{FL}$ - floresta de 57 anos; $\mathrm{CAP}$ - circunferência à altura do peito.

\begin{tabular}{|c|c|c|c|c|}
\hline \multirow[b]{2}{*}{ Espécies } & \multicolumn{4}{|c|}{ Número de indivíduos } \\
\hline & $\begin{array}{c}\text { RF } \\
\text { plântulas }\end{array}$ & $\begin{array}{c}\text { RF } \\
\text { juvenis }\end{array}$ & $\begin{array}{c}\text { FL } \\
\text { plântulas }\end{array}$ & $\begin{array}{c}\text { FL } \\
\text { juvenis }\end{array}$ \\
\hline Albizzia hasslerii (chodat) Burr & 82 & 23 & 37 & 57 \\
\hline Allophylus edulis Radlk. Ex warm & 10 & 37 & 0 & 1 \\
\hline Cecropia pachystachya Tréc. & 0 & 1 & 55 & 0 \\
\hline Celtis iguanaea (Jacq.) Sarg. & 0 & 0 & 35 & 9 \\
\hline Citrus limon (L.) Burm. F. & 0 & 0 & 0 & 2 \\
\hline Coussarea platyphylla M. Arg. & 3 & 105 & 0 & 4 \\
\hline Eugenia florida DC. & 0 & 22 & 0 & 10 \\
\hline Eugenia egensis DC. & 7 & 79 & 0 & 6 \\
\hline Eugenia hyemalis Cambess & 53 & 83 & 0 & 7 \\
\hline Garcynia gardneriana (Pl.et Tr.) & 3 & 90 & 0 & 0 \\
\hline Genipa americana L. & 0 & 6 & 0 & 14 \\
\hline Guarea macrophylla Vahl & 0 & 0 & 0 & 1 \\
\hline Inga vera Willd. & 9 & 60 & 0 & 3 \\
\hline Nectandra falcifolia(Nees.) Cast. Ex Mez. & 0 & 0 & 0 & 3 \\
\hline Nectandra molis (Kunth) Nees & 0 & 47 & 0 & 0 \\
\hline Ocotea diospyrifolia (Meissn.) Mez. & 0 & 12 & 0 & 1 \\
\hline Peltophorum dubium (Spreng) Taub & 0 & 4 & 0 & 0 \\
\hline Picramnia sellowii Planch & 0 & 0 & 18 & 79 \\
\hline Protium heptaphyllum & 0 & 57 & 0 & 0 \\
\hline Rollinia emarginata Schltdl & 0 & 0 & 0 & 3 \\
\hline Sapindus saponaria L. & 0 & 0 & 0 & 1 \\
\hline Senna alata (L.) Roxb. & 0 & 9 & 0 & 0 \\
\hline Sloanea garckeana k. Schum. & 0 & 0 & 0 & 4 \\
\hline Sloanea guianenses (Aubl.) Bentham. & 0 & 21 & 0 & 0 \\
\hline Spondias lutea $\mathrm{L}$. & 0 & 1 & 0 & 0 \\
\hline Tabernaemontana catharinensis A.DC. & 0 & 17 & 9 & 59 \\
\hline Trichilia pallida $\mathrm{Sw}$. & 1 & 56 & 0 & 12 \\
\hline Triplaris americana $\mathrm{L}$. & 0 & 6 & 14 & 103 \\
\hline Unonopsis lindmanii R.E.Fr. & 1 & 109 & 0 & 1 \\
\hline Zygia cauliflora (Willd.) Killip. & 0 & 8 & 0 & 0 \\
\hline TOTAL & 169 & 853 & 168 & 380 \\
\hline
\end{tabular}


dos indivíduos), e na classe II, Triplaris americana, Picramnia sellowii, Tabernaemontana catharinensis e Albizzia hasslerii ( $78 \%$ dos indivíduos).

O índice de diversidade de Shannon (H') foi de 1,61 nats para a classe I na FL e o índice de equabilidade de Pielou (J'), de 0,91. A classe II apresentou índice (H') de 2,1 nats e equabilidade (J'), de 0,68. O índice de Shannon (H') foi de 1,35 nats para a classe I no RFID, com equabilidade (J') de 0,63 , e para a classe II foi obtido índice ( $\mathrm{H}^{\prime}$ ) de 2,66 nats com equabilidade ( $\mathrm{J}$ ') de 0,86 . O valor de (H') da classe I foi significativamente superior na FL ( $\mathrm{p}=0,003)$, porém a classe II não apresentou diferença significativa para ( $\left.\mathrm{H}^{\prime}\right)$ entre as duas áreas $(\mathrm{p}=1,098)$.

As curvas de riqueza geradas por rarefação (Figura 2) indicaram que a riqueza de espécies entre as duas áreas diferiu somente para a classe I, com nível de riqueza mais elevado no RF. Ao considerar cada ambiente separadamente, observa-se que a riqueza diferiu entre as duas classes nos dois ambientes.

A análise de correspondência destendenciada (DCA), realizada com a classe I do estrato de regeneração arbóreo, não apontou agrupamentos específicos entre as duas áreas amostrais (Figura 3). Entretanto a DCA, gerada a partir da abundância das espécies da classe II, revelou a formação de dois agrupamentos, separando nitidamente as parcelas do RF daquelas da FL (Figura 4). O grupo relacionado às parcelas da FLT caracterizou-se pela abundância de Albizzia hasslerii, Celtis iguaneae, Genipa americana, Picramnia sellowii, Sloanea garckeana, Tabernaemontana catharinensis e Triplaris americana. $\mathrm{O}$ grupo com as parcelas do RF associou-se à abundância de Allophylus edulis, Coussarea platyphylla, Eugenia egensis, Eugenia hyemalis, Garcynia gardneriana, Eugenia florida, Inga vera, Ocotea diospyrifolia, Peltophorum dubium, Protium heptaphyllum, Senna alata, Sloanea guianenses, Trichilia pallida, Unonopsis lindmanii e Zygia cauliflora.

Os testes de correlação revelaram associações significativas entre a abundância das espécies e o tempo de inundação por parcela (Tabela 2). Dentre as espécies da classe I, Eugenia hyemalis apresentou correlação negativa e Celtis iguaneae apresentou correlação positiva com o tempo de inundação, as outras espécies não se mostraram correlacionadas com essa variável.

FIGURA 2: Curvas de rarefação geradas para as diferentes classes de tamanho nas duas áreas amostrais. RF - remanescente florestal; FL - floresta de 57 anos; CAP - circunferência à altura do peito.

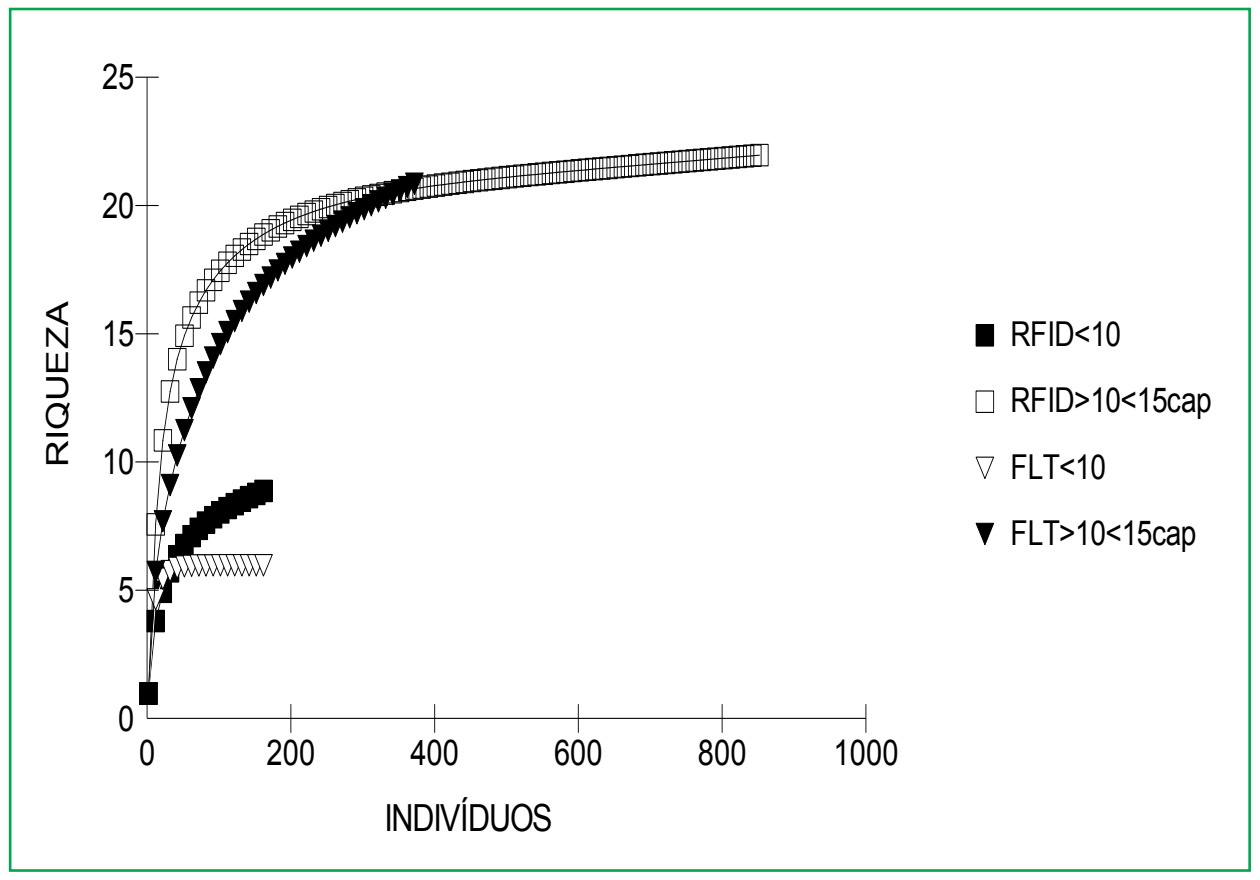


FIGURA 3: Análises de correspondência destendenciada (DCA) realizadas a partir da matriz de abundância por espécies da Classe I $-<$ $10 \mathrm{~cm}$ (plântulas), amostradas no RF (símbolos preenchidos) e na FL (símbolos sem preenchimento), na planície de inundação do alto Rio Paraná. As espécies são apresentadas através de suas letras iniciais.

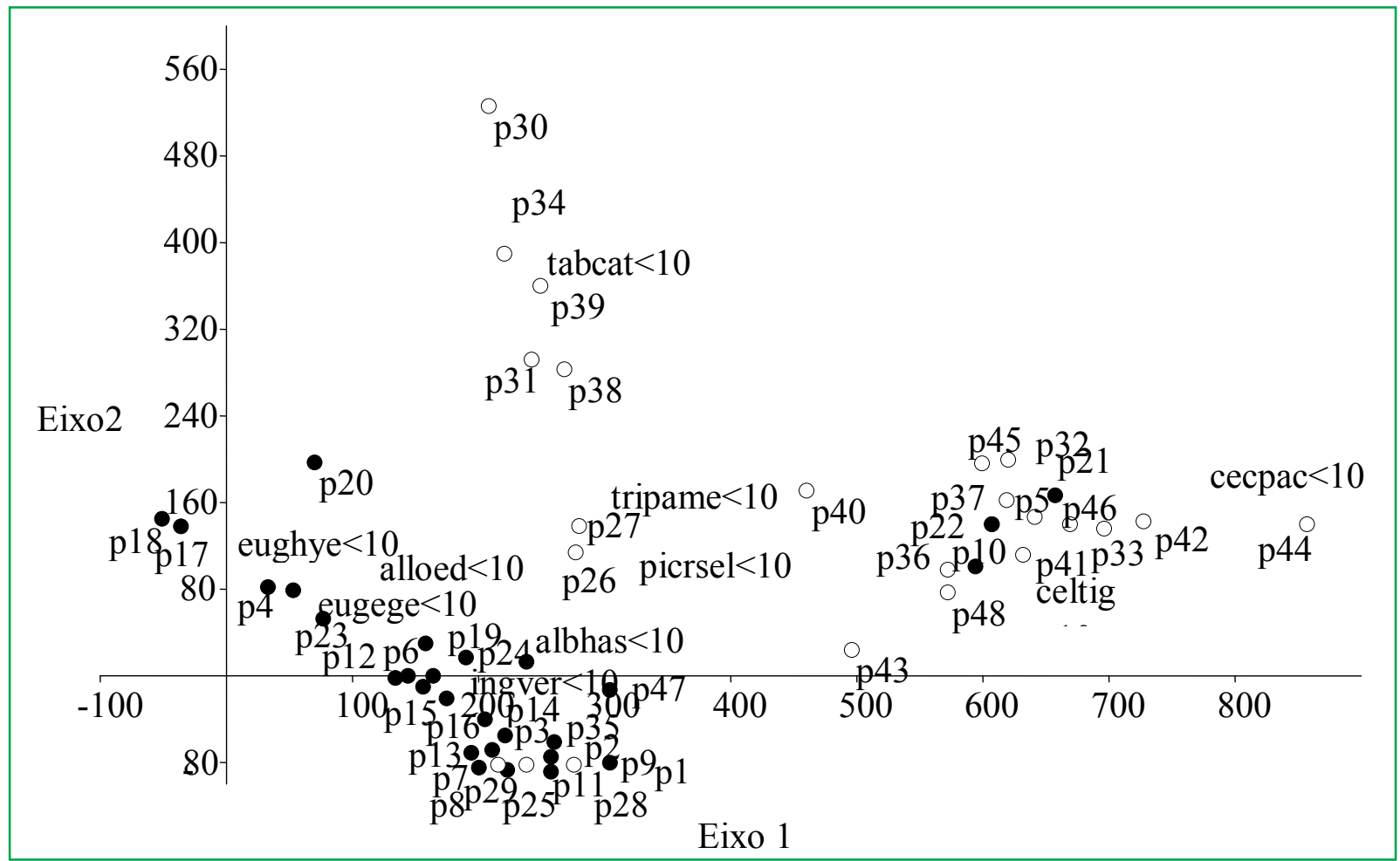

FIGURA 4: Análises de correspondência destendenciada (DCA) realizadas a partir da matriz de abundância por espécies da Classe II - > $10 \mathrm{~cm}$ e CAP $<15 \mathrm{~cm}$ (juvenis) amostradas no RF (símbolos preenchidos) e na FL (símbolos sem preenchimento), na planície de inundação do alto Rio Paraná. As espécies são apresentadas através de suas letras iniciais.

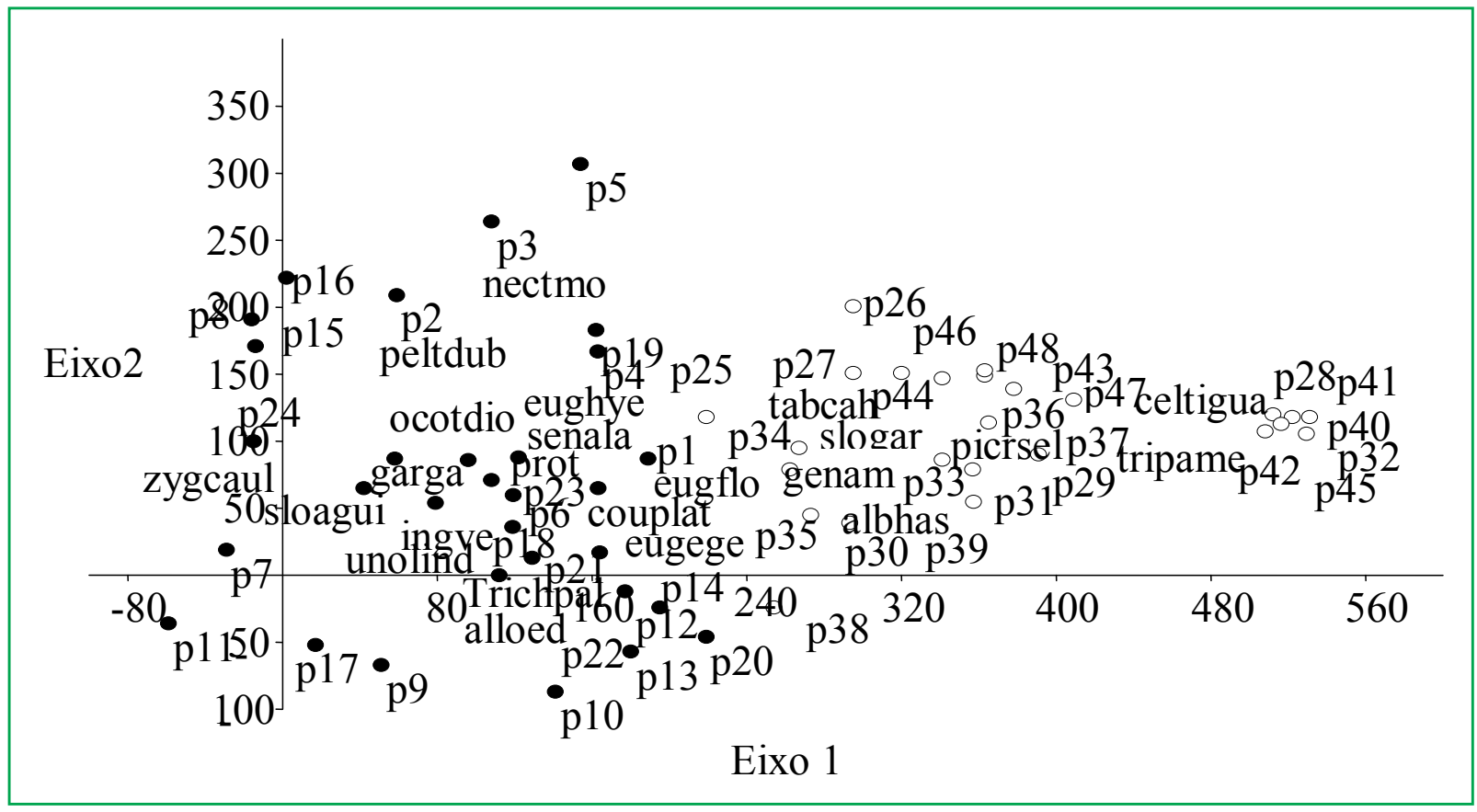


Das espécies da classe II, as que apresentaram correlação significativa foram, Tabernaemontana catharinensis, Triplaris americana, Picramnia sellowii e Sloanea garckeana, associadas positivamente ao tempo de inundação. Unonopsis lindmanii, Nectandra molis, Trichilia pallida, Garcynia gardneriana, Inga vera, Eugenia egensis e Protium heptaphyllum, foram negativamente associadas ao tempo de inundação

TABELA 2: Testes de correlação de Spearman (rs) realizados entre a abundância das espécies e o tempo de inundação por parcelas para as duas classes de tamanho, Classe I $-<10 \mathrm{~cm}$ e Classe II $->10 \mathrm{~cm}$ e CAP $<15 \mathrm{~cm}$, amostradas na planície de inundação do alto Rio Paraná.

\begin{tabular}{|c|c|c|c|}
\hline Espécies & rs & $\mathbf{t}$ & p \\
\hline Coussarea platyphylla & $-0,32$ & $-2,31$ & 0,025 \\
\hline Unonopsis lindmanii & $-0,78$ & $-8,56$ & $<0,001$ \\
\hline Sloanea guianenses & $-0,35$ & $-2,6$ & 0,012 \\
\hline Nectandra molis & $-0,43$ & $-3,26$ & 0,002 \\
\hline Trichilia pallida & $-0,42$ & $-3,13$ & 0,003 \\
\hline Garcynia gardneriana & $-0,74$ & $-7,53$ & $<0,001$ \\
\hline Tabernaemontana catharinensis & 0,38 & 2,76 & 0,008 \\
\hline Triplaris americana & 0,62 & 5,33 & $<0,001$ \\
\hline Ocotea diospyrifolia & $-0,29$ & $-2,02$ & 0,048 \\
\hline Inga vera & $-0,62$ & $-5,33$ & $<0,001$ \\
\hline Eugenia hyemalis & $-0,44$ & $-3,33$ & $<0,001$ \\
\hline Eugenia egensis & $-0,56$ & $-4,56$ & $<0,001$ \\
\hline Senna alata & $-0,31$ & $-2,18$ & 0,034 \\
\hline Protium heptaphyllum & $-0,66$ & $-5,94$ & $<0,001$ \\
\hline Albizzia hasslerii & 0,19 & 1,32 & 0,19 \\
\hline Allophylus edulis & $-0,36$ & $-2,65$ & 0,01 \\
\hline Zygia cauliflora & $-0,28$ & $-1,97$ & 0,054 \\
\hline Eugenia florida & $-0,12$ & $-0,79$ & 0,43 \\
\hline Picramnia sellowii & 0,68 & 6,25 & $<0,001$ \\
\hline Genipa americana & 0,12 & 0,8 & 0,42 \\
\hline Peltophorum dubium & $-0,27$ & $-1,88$ & 0,065 \\
\hline Sloanea garckeana & 0,37 & 2,73 & 0,009 \\
\hline Celtis iguanaea & 0,35 & 2,53 & 0,014 \\
\hline Albizzia hasslerii $<10 \mathrm{~cm}$ & $-0,33$ & $-2,35$ & 0,023 \\
\hline Inga vera $<10 \mathrm{~cm}$ & $-0,34$ & $-2,49$ & 0,016 \\
\hline Allophylus edulis $<10 \mathrm{~cm}$ & $-0,32$ & $-2,33$ & 0,024 \\
\hline Eugenia hyemalis $<10 \mathrm{~cm}$ & $-0,38$ & $-2,81$ & 0,007 \\
\hline Eugenia egensis $<10 \mathrm{~cm}$ & $-0,21$ & $-1,43$ & 0,158 \\
\hline Picramnia sellowii $<10 \mathrm{~cm}$ & 0,31 & 2,21 & 0,031 \\
\hline Triplaris americana $<10 \mathrm{~cm}$ & 0,17 & 1,16 & 0,25 \\
\hline T, catharinensis $<10 \mathrm{~cm}$ & 0,15 & 1,05 & 0,3 \\
\hline Celtis iguanaea $<10 \mathrm{~cm}$ & 0,58 & 4,79 & $<0,001$ \\
\hline Cecropia pachystachya $<10 \mathrm{~cm}$ & 0,24 & 1,66 & 0,104 \\
\hline
\end{tabular}




\section{Discussão}

O número de espécies amostradas nesse estudo (30 espécies no total) é uma quantidade de espécies relativamente pequena. No entanto, outros trabalhos como o desenvolvido por Budke et al. (2007), que considerando todos os indivíduos arbóreos com CAP $\geq$ $15 \mathrm{~cm}$, registrou um total de 30 espécies. DamascenoJunior et al. (2005), em trabalho realizado em uma floresta ribeirinha no Rio Paraguai, encontrou 37 espécies arbóreas, e um total de 22 espécies foram amostradas por Prata et al. (2011), em uma floresta ribeirinha no município de Rio Claro, levando-se em consideração todos os indivíduos arbóreos com $\mathrm{CAP} \geq 10 \mathrm{~cm}$. Embora o critério de inclusão dos indivíduos no processo de amostragem seja diferente entre os trabalhos citados, fica evidente a baixa riqueza de espécies arbóreas encontrada em ambientes sujeitos a inundações frequentes, mesmo considerando diferentes regiões geográficas.

A baixa riqueza de espécies observada em áreas inundáveis está diretamente relacionado ao estresse oriundo dos eventos de cheias. Condições de alta umidade inibem a germinação de sementes e limitam o estabelecimento dos indivíduos de muitas espécies arbóreas (LOBO; JOLY 2000). A ação das condições de saturação hídrica sobre a estrutura da comunidade vegetal se manifesta em função de alterações nas propriedades físico-químicas do solo, gerando um ambiente onde a disponibilidade de oxigênio é reduzida ou ausente (FELFILI et al., 2001), o pH do solo é modificado (NOGUEIRA; SCHIAVINI, 2003), e a concentração e ciclagem de nutrientes são profundamente alteradas (LOBO; JOLY, 2000; PARRON, 2004).

Tais condições, comuns em ambientes alagáveis, são fatores determinantes de ambientes altamente restritivos ao estabelecimento de muitas espécies arbóreas, resultando em comunidades vegetais nas quais se observa a predominância de poucas espécies com elevada abundância (ARAÚJO et al., 2004; BUDKE et al., 2008). Essa tendência é corroborada pelo presente estudo, no qual a baixa riqueza de espécies observada é acompanhada pela alta abundância de espécies como Albizzia hasslerii, Eugenia hyemalis, Celtis iguanaea, Picramnia sellowii, Unonopsis lindmanii, Coussarea platyphylla e Triplaris americana.
O estrato regenerante é um componente dos sistemas florestais que apresenta um comportamento dinâmico e é constituído por um conjunto de indivíduos que podem ser agrupados em uma variedade de classes de tamanho. Nesse estudo, as duas classes de tamanho consideradas diferiram nitidamente quanto ao número e composição de espécies, bem como, quanto ao número de indivíduos. Segundo Whitmore (1988), na fase de regeneração a densidade é influenciada pela dispersão e germinação das sementes, que ocorrem em maior quantidade próximo à árvore mãe. Durante essa fase de desenvolvimento as plântulas utilizam substâncias de reserva para sua manutenção, o que possibilita sua sobrevivência em uma ampla faixa de condições ambientais. No entanto, à medida que se desenvolvem, essas passam a obter recursos do ambiente (FELFILI et al., 2001), aumentando a competição entre os indivíduos, e limitações quanto à capacidade de sobrevivência e estabelecimento.

Dessa forma, apenas uma pequena proporção das sementes germinadas consegue se desenvolver e alcançar maior tamanho. Felfili (2000) registrou uma proporção de 30:9 entre indivíduos com menos de um metro de altura e aqueles com mais de um metro de altura e menos de cinco cm de diâmetro. De acordo com os resultados obtidos no presente estudo, foi observada uma maior quantidade de indivíduos na classe II, classe que envolve indivíduos de maior tamanho em relação à classe I. Como a classe I é representada por plântulas recém germinadas, sua abundância possivelmente está relacionada a eventos periódicos de chegada e germinação das sementes. Como constatado por Resende et al. (2003), muitas espécies apresentam períodos de baixa produção de sementes, seguido por um baixo recrutamento e passagem para maiores classes de tamanho.

Outro fator determinante da baixa abundância de indivíduos da classe I deve ser a ocorrência de inundações. As sementes de muitas espécies de florestas alagáveis perdem sua viabilidade após a submersão (FERREIRA; RIBEIRO, 2001), e mesmo aquelas já germinadas tendem a morrer por não tolerarem a submersão total. No entanto, à medida que se desenvolvem, muitas plantas adquirirem mecanismos morfológicos e fisiológicos que os tornam capazes de tolerar inundações parciais. 
Esse fato explica a maior abundância de indivíduos da classe II, que é representada por organismos mais desenvolvidos, e, consequentemente, capazes de sobreviver a inundações de curta duração.

Os resultados obtidos pela comparação entre os dois ambientes, demonstraram uma grande variação quanto ao número de indivíduos. Entretanto, não foi verificada diferença significativa quanto à riqueza e diversidade de espécies. Diversos trabalhos desenvolvidos em florestas alagáveis, têm encontrado maior riqueza e diversidade de espécies em áreas com menor suscetibilidade a inundações (FERREIRA; STOHLGREN, 1999; TEIXEIRA; ASSIS, 2005; SCARANO, 2006; BUDKE et al., 2007; UMETSU et al., 2011). Por isso, esperavase encontrar maior riqueza e diversidade no RF, que apresentou tempo de inundação significativamente inferior à FL. No entanto, essa expectativa não foi confirmada. Um caso ainda mais atípico reside no fato de se ter obtido maior diversidade no FL para a classe I. Esse resultado pode ser devido simplesmente à forte dominância de Albizzia hasslerii e Eugenia hyemalis no $\mathrm{RF}$, haja vista que nessa área observou-se maior riqueza de espécies na classe I.

Embora não se tenha observado diferença em termos de riqueza e diversidade entre as duas áreas, a composição de espécies apresentou diferenças. A DCA, realizadas com dados da classe I não foram capazes de discriminar grupos para cada área. Esse padrão certamente está relacionado à grande abundância de Albizzia hasslerii (única espécie comum às duas áreas para essa classe de tamanho), que se distribuiu por quase todas as parcelas das duas áreas amostrais. Em relação à classe II, observou-se a separação de dois grupos, formados por parcelas específicas de cada ambiente. Assim, fica evidente que a regeneração arbórea entre o RF e a FL pode estar assumindo trajetórias diferentes. Esses resultados estão de acordo com aqueles obtidos por (OLIVEIRA-FILHO et al., 1994; PINTO et al., 2005; PRATA et al., 2011), que observaram diferenças florísticas entre áreas com diferentes condições de umidade.

O grupo formado com as parcelas do FL se mostrou relacionado à abundância de Tabernaemontana catharinensis, Triplaris americana, Picramnia sellowii,
Sloanea garckeana e Celtis iguanaea, que se mostraram positivamente associados ao tempo de inundação das parcelas, o que demonstra que essas espécies apresentam preferência por locais com maior umidade. Por outro lado, o grupo formado pelas parcelas do RF, relacionouse principalmente à abundância de Unonopsis lindmanii, Nectandra molis, Garcynia gardneriana, Trichilia pallida, Inga vera, Eugenia hyemalis, Eugenia egensis e Protium heptaphyllum, que se associaram negativamente ao tempo de inundação, podendo ser caracterizadas como espécies típicas de locais mais secos.

Dessa forma, o regime de inundação diferenciado entre ambientes locais é capaz de gerar heterogeneidade ambiental que contribui para o incremento da diversidade de espécies em escala regional. Isso porque, determinadas espécies encontram-se melhor adaptadas a ambientes com maior tempo de inundação, ao passo que outras possuem hábitos preferenciais por ambientes com menor suscetibilidade a esses eventos. inundações. Essa força seletiva, exercida pelo regime de inundação pode influenciar no estabelecimento de plântulas e na sua passagem para maiores classes de tamanho.

\section{Referências}

ARAÚJO, M. M.; LONGHI, S. J.; BRENA, D. A.; BARROS, P. L. C.; FRANCO, S. Análise de agrupamento de um fragmento de floresta estacional decidual aluvial, Cachoeira do Sul, RS, Brasil. Ciência Florestal, Santa Maria, v. 14, n. 1, p. 133-147, 2004.

BIANCHINI, E.; GARCIA, C. C.; PIMENTA, J. A.; TOREZAN, J. M. D. Slope variation and population structure of tree species from different ecological groups in South Brazil. Anais da Academia Brasileira de Ciências, Rio de Janeiro, v. 82, n. 3, p. 643-652, 2010.

BUDKE, J. C.; JARENKOW, J. A.; OLIVEIRA FILHO, A. T. Relationships between tree component structure, topography and soils of a riverside forest, Rio Botucaraí, Southern Brazil. Plant Ecology, Dordrecht, v. 189, p. 187-200, 2007.

BUDKE, J. C.; JARENKOW, J. A.; OLIVEIRA FILHO, A. T. Tree community features of two stands of riverine forest under different flooding regimes in Southern Brazil. Flora, Jena, v. 203, p. 162174, 2008.

CAMPOS, J. B.; SOUZA, M. C. Arboreus vegetation of an alluvial riparian forest and their soil relations: Porto Rico island, Paraná River, Brazil. Brazilian Archieves of Biology and Technology, Curitiba, v. 45, p. 137-149, 2002.

CHAPIN, D. M.; BESCHTA, R. L.; SHEN, H. W. Relationships between flood frequencies and riparian plant communities in the upper Klamath Basin, Oregon. Journal of the American Water Resources Association, Middleburg, v. 38, p. 603-617, 2002. 
CHAPLA, T. E.; CAMPOS, J. B. Soil seed bank during succession at an abandoned pasture in the upper Paraná river-floodplain, Brazil. Acta Scientiarum Biological Sciences, Maringá, v. 33, n. 1, p. 59-69, 2011.

DALANESI, P. E.; OLIVEIRA-FILHO, A. T.; FONTES, M. A. Flora e estrutura do componente arbóreo da floresta do parque ecológico quedas do rio Bonito, Lavras, MG, e correlações entre a distribuição das espécies e variáveis ambientais. Acta Botanica Brasilica, Feira de Santana, v. 18, n. 4, p. 737-757, 2004.

DAMASCENO-JUNIOR, G. A.; SEMIR, J.; SANTOS, F. A. M.; LEITÃO FILHO, H. F. Structure, distribution of species and inundation in a riparian forest of Rio Paraguai, Pantanal, Brazil. Flora, Jena, v. 200, p. 119-135, 2005.

DURIGAN, G.; RODRIGUES, R. R.; SCHIAVINI, I. A heterogeneidade ambiental definindo a metodologia de amostragem da floresta ciliar. In: RODRIGUES, R. R.; LEITÃO FILHO, H. F. (Ed.). Matas ciliares: conservação e recuperação. São Paulo: Edusp, 2000. p. 159-167.

FELFILI, J. M. Dynamics of the natural regeneration in the Gama gallery forest in central Brazil. Forest Ecology and Management, Amsterdam, v. 91, p. 235-245, 1997.

FELFILI, J. M. Crescimento, recrutamento e mortalidade nas matas de galeria do planalto central. In: CONGRESSO NACIONAL DE BOTÂNICA, 51, 2000, Brasília. Palestras convidadas. Brasília: Embrapa Recursos Genéticos e Biotecnologia/Sociedade Brasileira de Botânica, 2000. p. 152-158.

FELFILI, J. M.; FRANCO, A. C.; FAGG, C. W.; SOUZA-SILVA, J. C. Desenvolvimento inicial de espécies de mata de galeria. In: RIBEIRO, J. F.; FONSECA, C. E. L.; SOUSA-SILVA, J. C. (Ed.). Cerrado: caracterização e recuperação de matas de galeria. Planaltina: Embrapa Cerrado, 2001. p. 779-811.

FERREIRA, J. N.; RIBEIRO, J. F. Ecologia da inundação em matas de galeria. In: RIBEIRO, J. F.; FONSECA, C. E. L.; SOUSASILVA, J. C. (Ed.). Cerrado: caracterização e recuperação de matas de galeria. Planaltina: Embrapa Cerrado, 2001. p. 425-451.

FERREIRA L. V.; STOHLGREN, T. J. Effects of river level fluctuation on plant species richness, diversity, and distribution in a floodplain forest in Central Amazonia. Oecologia, Berlin, v. 120, n. 4, p. 582-587, 1999.

GIEHL, E. L. H.; JARENKOW, J. A. Gradiente estrutural no componente arbóreo e relação com inundações em uma floresta ribeirinha, rio Uruguai, sul do Brasil. Acta Botanica Brasilica, Feira de Santana, v. 22, n. 3, p. 741-753, 2008.

JUNK, W. J. The flood pulse concept of large rivers: learning from the tropics. Archiv für Hydrobiologie, Suppl., Stuttgart, v. 115, n. 3, p. 261-280, 1999.

LOBO, P. C.; JOLY, C. A. Aspectos ecofisiológicos da vegetação de mata ciliar do Sudeste do Brasil. In: RODRIGUES, R. R.; LEITÃO FILHO, H. F. (Ed.). Matas ciliares: conservação e recuperação. São Paulo: Edusp, 2000. p. 143-157.

MAACK, R. Geografia física do Estado do Paraná. Curitiba: Imprensa Oficial do Paraná. 2002. 350 p.

MARQUES, M. C. M. Dinâmica da dispersão de sementes e regeneração de plantas da planície litorânea da Ilha do Mel - PR. 2002. 145 f. Tese (Doutorado em Biologia Vegetal) Universidade Estadual de Campinas, Campinas. 2002.
NEIFF, J. J.; IRIONDO, M. H.; CARIGNAN, R. Large Tropical South American Wetlands: An Overview. Proc. of the Internat. Workshop on the Ecology and Management of Aquatic Terrestrial Ecotones: 1994. p. 156-165.

NILSSON, C.; SVEDMARK, M. Basic principles and ecological consequences of changing water regimes: Riparian plants communities. Environmental Management, New York, v. 30, n. 4, p. 468-480, 2002.

NUPELIA- Núcleo de pesquisas em Limnologia, Ictiologia e aquicultura. Nível fluviométrico da planície de inundação do alto Rio Paraná. 2012. Disponível em: < http://www.peld.uem.br/peldnivel_fluviometrico. html >. Acesso em: 10 maio 2012.

NOGUEIRA, M. F.; SCHIAVINI, I. Composição florística e estrutura da comunidade arbórea de uma mata de galeria inundável em Uberlândia, MG, Brasil. Bioscience Journal, Uberlândia, v. 19, p. 89-98, 2003.

OLIVEIRA, E. C. L.; FELFILI, J. M. Estrutura e dinâmica da regeneração natural de uma mata de galeria no DF. Brasil. Acta Botanica Brasilica, Feira de Santana, v. 19, n. 4, p. 801-811, 2005. OLIVEIRA-FILHO, A. T.; VILELA, E. A.; CARVALHO, D. A.; GAVILANES, M. L. Effects of soils and topography on the distribution of tree species in a tropical riverine forest in southeastern. Brazil. Journal of Tropical Ecology, Winchelsea, v. 10, p. 483-508, 1994.

PARRON, L.M. Aspectos da ciclagem de nutrientes em função do gradiente topográfico em uma mata de galeria no DF. 2004. 181 f. Tese (Doutorado em Ecologia) - Universidade de Brasília, Brasília. 2004.

PINTO, J. R. R.; OLIVEIRA- FILHO, A. T.; HAY, J. D. V. Influence of soil and topography on the composition of a tree community in a central Brazilian Valley forest. Edinburgh Journal of Botany, Edinburgh, v. 62, p. 69-90, 2005.

PRATA, E. M. B.; PINTO, S. A. F.; ASSIS, M. A. Fitossociologia e distribuição de espécies arbóreas em uma floresta ribeirinha secundária no Município de Rio Claro, SP, Brasil. Revista Brasilileira de Botânica, São Paulo, v. 34, n. 2, p. 159-168, 2011.

RESENDE, J. C. F.; KLINK, C. A.; SCHIANINI, I. Spatial heterogeneity and its influence on Copaifera langsdorffii Desf. (Caesalpiniaceae). Brazilian Archieves of Biology and Technology, Curitiba, v. 46, n. 3, p. 405-414, 2003.

RODRIGUES, R. R. Florestas ciliares? Uma discussão nomenclatural das formações ciliares. In: RODRIGUES, R. R.; LEITÃO-FILHO, H. F. (Ed.). Matas ciliares: conservação e recuperação. São Paulo: Edusp, 2001. p. 91- 99.

RONDON NETO, R. M. Estudo da regeneração natural e aspectos silviculturiais de uma clareira de formação antrópica. 1999. 122 f. Dissertação (Mestrado em Ciências Florestais) Universidade Federal de Lavras, Lavras. 1999.

SCARANO, F.R. Plant community structure and function in a swamp forest within the Atlantic rain forest complex: a synthesis. Rodriguésia, Rio de Janeiro, v. 57, p. 491-502, 2006.

TEIXEIRA, A. P.; ASSIS, M. A. Caracterização florística e fitossociológica do componente arbustivo-arbóreo de uma floresta paludosa no Município de Rio Claro (SP), Brasil. Revista Brasileira de Botânica, São Paulo, v. 28, p. 467-476, 2005.

TEIXEIRA, A. P.; ASSIS, M. A. Relação entre heterogeneidade ambiental e distribuição de espécies em uma floresta paludosa no 
Município de Cristais Paulista, SP, Brasil. Acta Botanica Brasilica, Feira de Santana, v. 23, n. 3, p. 843-85, 2009.

TEIXEIRA, A. P.; ASSIS, M. A.; SIQUEIRA, F. R.; CASAGRANDE, J. C. Tree species composition and environmental relationships in a Neotropical swamp forest in Southeastern Brazil. Wetlands Ecology and Management, Baarn, v. 16, p. 451-461, 2008 .
UMETSU, R. K.; GIRARD, P.; MATOS, D. M. S.; SILVA, C. J. Efeito da inundação lateral sobre a distribuição da vegetação ripária em um trecho do rio cuiabá, MT. Revista Árvore, Viçosa, v. 35, n. 5, p. 1077-1087, 2011.

WHITMORE, T. C. The influence of tree populations dynamics on forest species composition. In: DAVY, A. J.; HUTCHINGS, M. J.; WATKINSON, A. R. (Ed.). Plant population ecology. London: Blackwell Scientific Publication, 1988. p. 271-291. 